\title{
History of Development and Regulations for Regenerative Medicines in Japan
}

\author{
Hanayuki Okura and Akifumi Matsuyama* \\ National Institutes of Biomedical Innovation, Health and Nutrition, Osaka, Japan
}

\begin{abstract}
Regenerative medicinal products were dealt with fewer than two categories only, pharmaceutical products and medical devices in the previous Pharmaceutical Affairs Law (PAL). Because regenerative medicinal products could not fully fit within the conventional regulatory contexts for pharmaceuticals, they were less available to the public. To overcome this issue, the Japanese parliament has legislated Laws for the Promotion of Comprehensive Measures to Facilitate Swift Distribution and Safe Use of Regenerative Medicines available to the Public (Regenerative Medicine Promotion Act). The Japanese government has changed the regulatory framework for regenerative medicinal products, 1) upgrading of directives for human stem cell-based clinical research to the Law concerning the Establishment of Safety of Regenerative Medicine Materials (Regenerative Medicine Safety Assurance Act), and 2) setting a new category for regenerative medicinal products in the Pharmaceutical and Medical Device Act (PMD Act), the latest revised PAL. It is noteworthy that the PMD Act permits expedited, conditional, and time-limited marketing approval as an exception for non-homogeneous regenerative medicinal products when efficacy is anticipated while safety is demonstrated. With these revisions of the regulatory framework for regenerative medicinal products, two tracks have become available for societal contribution to regenerative medicines. Japan is the only country where society has these two tracks, and social experiments on regenerative medicines in spectacular proportion are launched for societal contribution. We review here the history of the struggle to accelerate the availability of regenerative medicinal products for patients in Japan.
\end{abstract}

Keywords: Regulatory framework; Regenerative medicine promotion act; PMD act; Regenerative medicinal products; Japan; Legislations

\section{Introduction}

In relation to the enforcement of Laws for the Promotion of Comprehensive Measures to Facilitate Swift Distribution and Safe Use of Regenerative Medicines available to the Public (Regenerative Medicine Promotion Act) 1 established in April 2013 and enacted in May 2013, the Law concerning the Establishment of Safety of Regenerative Medicine Materials (Regenerative Medicine Safety Assurance Act), 2 and the Pharmaceutical Affairs Law (currently Pharmaceutical and Medical Device Act: PMD act) 3 were revised (the new regulation was established in November 2013 and enacted on November 25, 2014). The Regenerative Medicine Safety Assurance Act was intended to integrate into a single regulatory scope for legislation the techniques on regenerative medicine conventionally used in separate areas of clinical research (regulated by rules on human stem cell guidance) and medical services outside the scope of the national health insurance scheme (no previous regulations). The scope of the regulation includes regenerative medicines provided as part of clinical research or medical care (including uninsured medical services). The law also structures systems to capture the status of research/practices and to secure the safety of regenerative medicines. The revised Pharmaceutical Affairs Law (PAL) included regenerative medicine products as a new category in addition to conventional pharmaceutical products and medical devices, it clearly provides description of a conditional approval system with limited validity depending on the properties (system to accelerate an approval) and mandates informed consent procedures to explain potential risks to patients and thereby to obtain consent and document preparation and retention of post-marketing surveys, sales, and use as an effort to secure safety. In this section, the paper primarily describes the Regenerative Medicine Safety Assurance Act as well as the revised PAL (Pharmaceutical and Medical Device Act), and provides a framework for regenerative medicine regulation in Japan [1-4].

\section{History of Establishment of Three Regenerative Medicine-Related Acts}

The Japanese population healthy-aged and/or diseased have been eager for regenerative therapy, which could be applied to intractable diseases, but they wondered whether unapproved cell therapy in private clinics should lead to serious adverse outcomes. To meet to their desires, Japanese government has launched "A five-year Medical Innovation Strategy" in June 2012 and "the Japan Recovery Strategy in July 2012". In response to these initiatives, the Health Sciences Council Scientific Technology Panel: Subcommittee for Promotion and Assurance for Safety of Regenerative Medicines was set up on August 20,2012, and the first meeting of the expert committee was held in September 4, 2012. In the initial movement towards the legislation of the present act, the direction was not clear as to whether human stem cell clinical research guidance would be revised or legislation would take place and the coordination was to be completed by the summer in 2013. In the awake of the Nobel Prize awarded to Professor Shinya Yamanaka on October 8, 2012, voluntary members of the Diet (Parliament) from three political parties agreed on October 23 to submit the proposed Regenerative Medicine Promotion Act in the next

*Corresponding author: Akifumi Matsuyama, MD, PhD, Director, Platform of Therapeutics of Rare Disease, National Institute of Biomedical Innovation, National Institutes of Biomedical Innovation, Health and Nutrition., 7-6-8, Saito Asagi, Ibaraki-city, Osaka 567-0085, Japan, Tel: +81-72-641-9899; Fax: +81-72-6419829; E-mail: akifumi-matsuyama@umin.ac.jp

Received November 14, 2016; Accepted December 28, 2016; Published January 05, 2017

Citation: Okura H, Matsuyama A (2017) History of Development and Regulations for Regenerative Medicines in Japan. J Stem Cell Res Ther 6: 372. doi: 10.4172/2157-7633.1000372

Copyright: (C) 2017 Okura H, et al. This is an open-access article distributed under the terms of the Creative Commons Attribution License, which permits unrestricted use, distribution, and reproduction in any medium, provided the original author and source are credited. 
ordinary Diet session and discussed the submission of separate laws for regenerative medicine in the ordinary Diet session for the fiscal year of 2013. These decisions eventually accelerated the discussion not only on the guideline, but also for the direction towards legislations in the second meeting in November. The third meeting in December confirmed the legislative direction, and consequently the report "Structuring the Framework for Securing the Safety and Promotion of Regenerative Medicine" was drawn up and reviewed through the seventh discussions in April 2013, and the proposed Regenerative Medicine Safety Assurance Act was submitted in May 2013 together with the Revision of the Pharmaceutical Affairs Law (PAL). The bill was unanimously passed by the Diet. The Regenerative Medicine Safety Assurance Act and PAL revision were established and enacted on the same day, which can be translated into an explicit message indicating the regulatory process on regenerative medicine will go through these two legislative paths for some time [2].

\section{The Regenerative Medicine Promotion Act}

The Law for the Promotion of Comprehensive Measures to Facilitate Swift Distribution and Safe Use of Regenerative Medicines available to the Public (Regenerative Medicine Promotion Act) was submitted to the Diet as a bipartisan lawmaker-initiated bill. The legislation was then approved. The aim of the legislation is to ensure that the public is provided with access to regenerative medicine in a swift and safe manner, which was enabled by establishment of the basic concepts for promoting regenerative medicine research/development, supply and distribution, by identifying the responsibilities of the Japanese government, medical practitioners, researchers, and business entities and by facilitating the comprehensive promotion of measures from research and development to the commercialization of regenerative medicine, thereby contributing to improvement of quality of medical services available for the public, health and hygiene [2-10].

With regard to the responsibilities to promote regenerative medicine, the Japanese government is required to develop and implement comprehensive measures, provide education to the public, establish relevant ministerial collaborative structures, and announce basic directions on research/development and promotion of swift and safe supply and distribution of regenerative medicines. Medical practitioners and researchers are required to cooperate with measures implemented by the Japanese government whereas business entities are expected to collaborate with such governmental measures.

The following are the schemes expected to be implemented by the Japanese government:

\section{Legislative measures.}

2. Promoting research and development of innovative regenerative medicine.

\section{Organization of regenerative medicine environment.}

4. Organization of clinical research environment.

5. Streamlining the structure for reviewing regenerative medicine products.

6. Promotion of regenerative medicine business.

\section{Securing human resources.}

The establishment of the present law is a historic milestone where the Regenerative Medicine Safety Assurance Act was legislated to legally endorse regenerative cell therapy technology as medical practices provided under the Medical Practitioner Act/Medical Service Act as the regenerative medicine-related law. A new chapter for regenerative medicine products was also included as regenerative/cell therapy in PAL revision (PMD Act) and incorporated as part of the revision [4-8].

\section{The PMD Act (Revised PAL)}

\section{History}

The overview of the PAL revision in 2012 (Law pertaining to Ensuring Quality, Efficacy and Safety of Pharmaceutical Products, Medical Devices: Pharmaceutical and Medical Device Act or PMD Act), envisioned the institution of required measures to ensure the safe and swift supply of pharmaceutical products and medical devices and to mandate for the first time the regulatory submission of package inserts, expanded the scope of certification by registered notified bodies for medical devices, and established a system for conditional and timelimited approvals for regenerative medicine products among others. These were to be legislated in a cabinet order within the prescribed days not exceeding one year after the promulgation of the revision. The revision was enacted on November 25, 2014.

\section{Overview of PAL}

Conventionally, pharmaceutical products to be manufactured and marketed are subject to regulations on manufacturing and marketing businesses for such products. In contrast, the PMD Act was initially intended to regulate continuously and repeatedly licenses for manufacturing/marketing pharmaceutical products as business entities so as to assure the fulfilment of three requirements of efficacy, safety, and quality. However, in alignment with changes in the socioeconomic situations, such as international consistency for the regulatory system, development of scientific technology, and diversified corporate activities, the major revision in 2002 enhanced post-marketing safety measures and transformed the license approval system to wholesaler distribution system from the view that safety assurance of the distributed products is the essential component. This means that regulations were tightened to ensure safe and effective use of products, and more focus was placed on follow-up primarily on distribution of products and post-marketing adverse drug reactions [11-14].

Especially in the case of regenerative medicines, a new definition was established for regenerative medicine products, and new regulations/systems were established based on the properties of regenerative medicine products by respecting discussions that identify the importance of achieving safety and reassurance by securing the safety and ethics in light of the properties of regenerative medicines different from conventional pharmaceutical products/medical devices in order to implement measures for smooth promotion of swift commercialization in response to development of regenerative medicine research. Regenerative medicine products include not only cell preparations and ex-vivo gene therapy, but also gene therapies, different from the Regenerative Medicine Safety Assurance Act as this paper will describe later. To easily capture the range of regenerative medicine products in comparison with US/EU regulation counterparts, the range equates comparatively in the US system to the biologics covered by the Center for Biologics Evaluation and Research (CBER), excluding protein drug products, therapeutic antibodies, and nucleic acid medicine, whereas the range is covered by so-called ATMP (Advanced Therapy Medicinal Products) of the European regulation.

It is noteworthy that the new law permits expedited, conditional, and time-limited marketing approval as an exception for inhomogeneous regenerative medicine products when efficacy is anticipated while safety is demonstrated. Although an exceptionally expedited conditional 
approval system was already stipulated in the previous PAL, the new law now clearly stipulates the approval as the establishment of a new category as regenerative medicine products. Ambiguity remains as to whether non-homogenous regenerative medicine products indicate autologous cell preparation or whether regenerative medicine products should be categorized as non-homogenous. Moreover, controversy remains as to how to predict the efficacy and how it should be interpreted and whether this efficacy decision should apply when some verified effective response is reported among many patients. So far, no QA notice has been issued for an effort to clarify these varying interpretations, which leaves out the possibility that future regulatory reviews may delineate the interpretations. Exceptional early approvals will involve verification of efficacy and safety as an effort for postapproval activities, and when post-approval efficacy/safety evaluations equivalent to re-examination as in the case of pharmaceutical products may fail to demonstrate the predicted benefits, the exceptionally expedited approval will be revoked.

On pharmaceutical regulation, pharmaceutical products/medical devices will receive regulatory approval if secured for the quality/ efficacy/safety in light of scientific technology level at the time of clinical trial notification, clinical study, and approval application. The products/devices will be listed in national health insurance drug price or reimbursement price, and then the products/devices will be marketed in accordance with the health insurance law.

In general, pharmaceutical products/medical devices are commercialized through clinical trial notification, clinical studies, and ultimately applications for regulatory approval. The safety and quality of the products under the approved application need to observe the standards for basic document preparation, a Ministerial Notification No. 1314 issued by Pharmaceutical and Food Safety Bureau (PFSB) of the Ministry of Health, Labour and Welfare (MHLW) in 2000 Attachments 1 and 2 revision notices (5 guidelines issued in 2012). Attachment 1 of the Notification No. 1314 issued by the Pharmaceutical and Food Safety Bureau (PFSB) of the Ministry of Health, Labour and Welfare (MHLW) in 2000 is regarded as equivalent to the socalled GTP, which specifies the concept of public hygiene to prevent the spread of infectious diseases mediated by cellular tissue-based pharmaceutical products. The 5-guidance issued in 2012 is intended to prevent undesirable changes/denaturalization of shapes/quality during the manufacturing process of regenerative medicine products and thereby secure the quality/efficacy/safety as pharmaceutical products/ medical devices.

In the revised PAL (PMD act), the manufacturing of regenerative medicine products is required to observe the GCTP (Good Gene, Cellular, and Tissue-based Manufacturing Practice) instead of the GMP. The introduction of the practice reflects the fact that sterilization is not possible as in the case of low-molecular pharmaceutical products and the view that manufacturing control for regenerative medicines requires careful management. It is noteworthy that quality risk management was incorporated as active efforts for assessment and control in accordance with appropriate procedures for product quality risks over the entire duration from the early stage of development to the completion of the manufacturing process and marketing, and thereby to promote continuous improvements of product manufacturing procedures and product quality [14-18].

\section{Regulatory strategic consultation}

Ideally, regulatory strategic consultation should be utilized for the regulatory (medical device) approval of regenerative medicine products. The regulatory strategic consultation was transformed from the confirmatory application system initiated in 1999.

Regulatory strategic consultations towards the initiation of clinical studies should be sought for, preferably in the order of consultations on quality, non-clinical safety, and clinical study protocols. Quality consultation verifies that the manufacturing and formulation process and the raw materials do not contravene the relevant regulations and then quality variables will be decided in an official consultation meeting. In absolute essence, the use of raw material must involve conformity with the standards for raw materials and verification of the entire tissues. Currently, many specimens to be used in regenerative medicine research must be proved to comply with the standards for raw materials, though most of the cases fall short of achieving conformity with the raw material standards. On the other hand, the non-clinical safety consultation involves discussion of the entire tissues for which the disclosure becomes unavoidable at that stage. Quality variables should be decided in view of three aspects; identification, efficacy, and safety of the cells in the final drug products. Cell surface markers may be useful in cell identification while their use may fall short of providing rational persuasion in terms of efficacy and safety variables.

After quality consultation, the non-clinical safety consultation will discuss whether first-in-man use is reasonably feasible. It should be remembered that unless the manufacturing formulation process and quality variables are fully established, all the studies might have to be redone, which deserves particular attention. The redo is warranted because even slight variation of the manufacturing and formulation process no longer assures equivalence to the properties of the cells previously validated for safety, and any changes to quality variables do not provide any safety assurance of quality comparability. Non-clinical safety consultations may discuss residual toxicities of low molecular compounds used for inducing differentiation.

Clinical study protocol consultations will involve discussions on protocol and design decisions to validate the safety or efficacy based on non-clinical trial results. In particular, it is like that target disease and subject inclusion criteria are decided from a non-clinical efficacy study (non-clinical Proof-of-Concept (POC) study) while subject exclusion criteria will be decided from non-clinical safety study results. Some of the issues that need to be particularly focused on in non-clinical POC studies include the appropriateness of model animals, disease extrapolation to humans, and links among the variables in the model animals and ones in clinical studies defined as endpoints. Insufficient non-clinical POC study designs would undermine the possibility of proceeding to a clinical study. Still, clinical use became possible even without POC after the establishment of the Regenerative Medicine Safety Assurance Act. In such case, the development stage will move to advanced medical care instead of a clinical study, and then an advanced medical care assessment panel will decide whether products can be covered by insurance for medical services [18-20].

\section{Regenerative Medicine Safety Assurance Act}

\section{History}

In relation to the establishment of the Regenerative Medicine Promotion Act, the Law pertaining to the assurance of Regenerative Medicine Safety (Regenerative Medicine Safety Assurance Act) was established as part of the Japanese government's duty to promote legislative treatment for regenerative medicines. The law is intended to clarify measures related to consideration of safety assurance and life ethics on the techniques to be used in regenerative medicines and other measures to be instituted by the suppliers of regenerative medicines, 
to define the system related to manufacturing license for specified cell processing products in order to facilitate swift and safe supply and distribution of regenerative medicines, and thereby to contribute to the improvement of medical services and health care quality (Article 1). The distinction from the PAL is that the products are treated as part of medical technology provided under the Medical Practitioner Act/ Medical Service Law, which does not overlap PAL regulations. The idea that the law is a successor of the spirit of the Directives for Human Stem Cell-based Clinical Research as regulatory measures would facilitate an understanding of the law. Consequently, the directives were abolished in connection with the enactment of the present law. One of the backgrounds to the establishment of the present law relates to a national fatality caused by a South Korean biotech company, RNL Bio. The fact that the term regenerative medicine is also used in the plastic and reconstructive surgery field necessitated the registration of all medical institutions engaged in providing regenerative medicines.

This chapter will briefly touch on the history of the Regenerative Medicine Safety Assurance Act that succeeded the regulatory intent of its predecessor, the Directives for Human Stem Cell-based Clinical Research. The directives were first formulated and issued as a regulatory guidance pertaining to regenerative medicine under MHLW Notification No. 425 for 2006. The directives were entirely revised by another Ministerial Notification No. 380 for 2010, which recognized iPS cell-based regenerative medicine research. In 2013, the conditional use of ES cell-based regenerative medicine was permitted by Ministerial Notification No. 317. Originally, the principal investigators were required to wait for reviews on relevant clinical research protocols by ethical and other committees established in their research organizations and the approval by the head of the research organizations, then seek some ministerial advice based on the Directives for Human Stem Cellbased Clinical Research, before launching regenerative medicine-based clinical research. Naturally at this stage, such practices were deemed special medical care, which contravened Article 18 of the regulations on insurance medical institutes and health care long-term care and were outside the scope of insurance reimbursement, including the cost covered as general medical practices. This is because, even if explanations were provided and consent was obtained, a probable difference can strongly exist in understanding between the medical practitioners and patients and an unfair burden from such difference on patients should be prevented, and any medical practices not validated for efficacy/safety should be avoided.

\section{Overview of regenerative medicine safety assurance act}

The Regenerative Medicine Safety Assurance Act sets two major cornerstones: one is the establishment of mandatory adherence to the Regenerative Medicine Supply Standard (an ordinance) and penal codes for failed adherence to ministerial orders, including suspension/ improvement orders, and the other is the involvement of a preliminary review system by the regenerative medicine committee. The regenerative medicine supply standards are specified in article 3 of the law. Specifically, the supply must adhere to ordinances consigned by articles 42 and 44 (i.e. articles 42 and 44 standards). Moreover, article 3 of the law provides classifications of regenerative medicines into three categories of Types 1-3 regenerative medicines, based on the level of impacts on human life and health, with the necessary procedures. To facilitate the understanding of the classification, Type 1 regenerative medicines represent a class of products derived from homologous cells and pluripotent stem cells or similar cells, while Type 3 regenerative medicines cover products intended for cancer immune therapy and those involving minimal manipulations. The idea is illustrated as a flowchart, available as the QA notice (Figure 1). In the discussion of the legislation process, the categorical instrument was reviewed for its scientific rationality/ethics and was understood as a risk classification for risk control. Specifically, Type 1 representing iPS cell-based regenerative medicines (research) can potentially involve unpredictable risks requiring sufficient levels of discussions and thus require a central review. Type 2 products are those for which controlling risks have been identified as challenges in light of experiences from human stem cell clinical research directive reviews and thus review by specifically certified regenerative medicine review committee will suffice. The Type 3 category requires discussion by the Certified Regenerative Medicine Review Committee, an internal ethical committee within an organization due to non-significant controlling risk level [18].

The procedures related to the supply of regenerative medicines are shown in Figure 2. The supply scheme for Type 1 products involves hearing of opinions of the Specially Certified Regenerative Medicines Committee (Ethical Review Board) and then notification in writing to the MHLW minister of such supply. The scheme has 90-day inwait restriction, during which the Health Minister seeks the opinion of Health Sciences Council to verify safety. If the product fails to meet the standards for safety, the Minister is empowered to order a scheme change. Noteworthy points are (1) review by specially certified regenerative medicines committee, (2) submission of regenerative medicines supply scheme to the Health Minister, and (3) supply initiation after 90-day restriction (for central review by the Health Sciences Council Regenerative Medicines Review Panel), which are not substantially different from the review required under the Directives for human stem cell-based Clinical Research. The supply of Type 1 products is practically under a permission-based scheme. The supply scheme of Type 2 regenerative medicines involves seeking the opinion of the Specially Certified Regenerative Medicines Committee and then reports in writing to the MHLW Minister of the product supply. Type 2 scheme entails the above (1) and (2) and thus relaxes the conventional double-layer review and practically under a notification-based scheme. Meanwhile, the quality assurance of the Specially Certified Regenerative Medicines Committee poses immediate challenge, and in fact, the reality is that inadequate committees will eventually fade out. The Type 3 product supply scheme requires seeking opinions from the Certified Regenerative Medicines Committee and then reports in writing to the MHLW Minister of such supply. The Certified Regenerative Medicines Committee can be set up by individual medical institution and consequently the levels can evidently vary. Whether appropriate reviews are done can dictate the future of regenerative medicines. Specially Certified Regenerative Medicines Committees are defined as expert panels of highly competent review capability and independence, and thus not all medical institutions can set up the committee. The committee is managed in a manner similar to that of the central IRB and will have to undertake remunerated reviews.

For measures that ensure the proper supply of regenerative medicines, measures for informed consent and privacy information protection are defined, and adverse events should be immediately reported to the MHLW Minister, and the Minister should seek opinions from the Health Sciences Council for necessary measures, offering consideration for the fact that regenerative medicines are not fully established within the health care system. The MHLW Minister is empowered to issue an order for improvement of safety assurance, and when the order is violated, the Minister can impose restrictions on the supply of regenerative medicines, as well as temporary measures to suspend the supply of regenerative medicine when warranted in order to prevent the spread of damage for health care reasons. The 
Citation: Okura H, Matsuyama A (2017) History of Development and Regulations for Regenerative Medicines in Japan. J Stem Cell Res Ther 6: 372. doi: $10.4172 / 2157-7633.1000372$

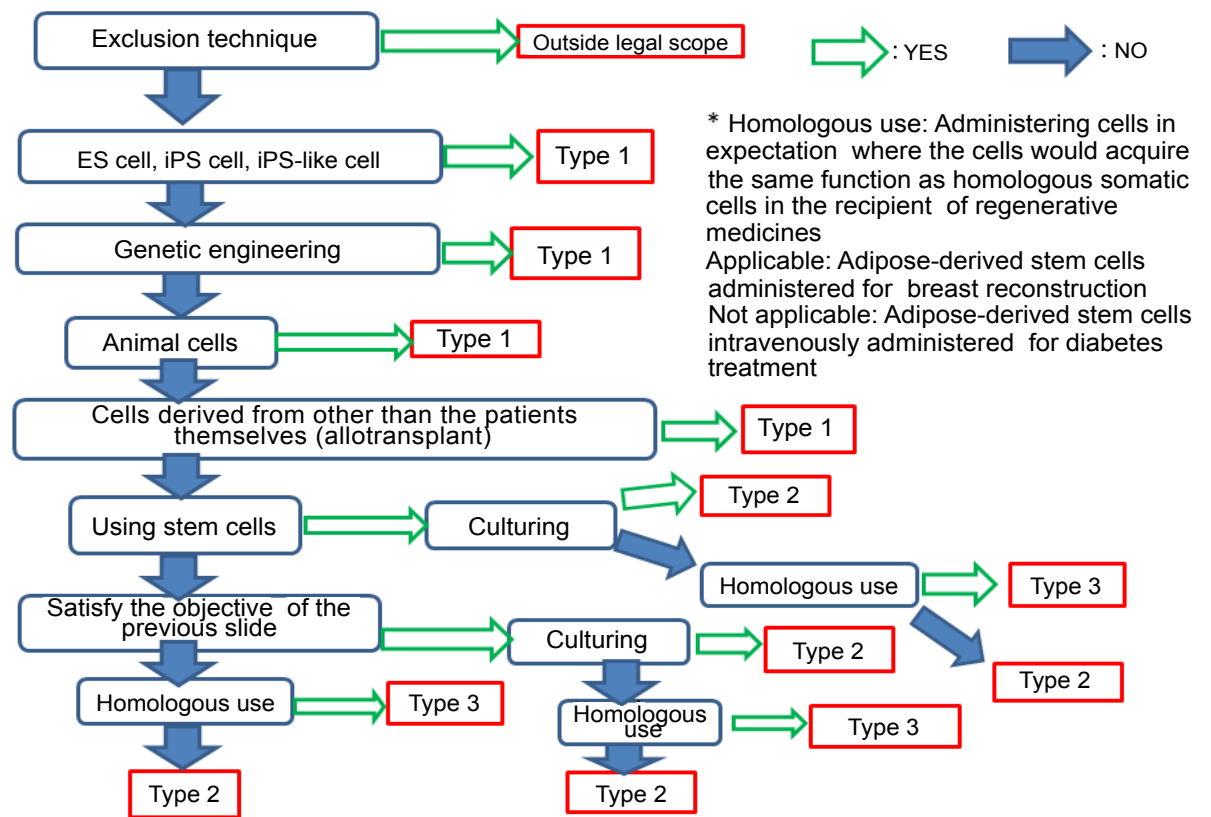

Figure 1: Risk classification of techniques for Type 1-3 regenerative medicines. The regenerative medicinal products are categorized into three groups according to their risk assessment in the Regenerative Medicine Safety Assurance Act. ES: Embryonic Stem Cells, iPS: Induced Pluripotent Stem Cells.

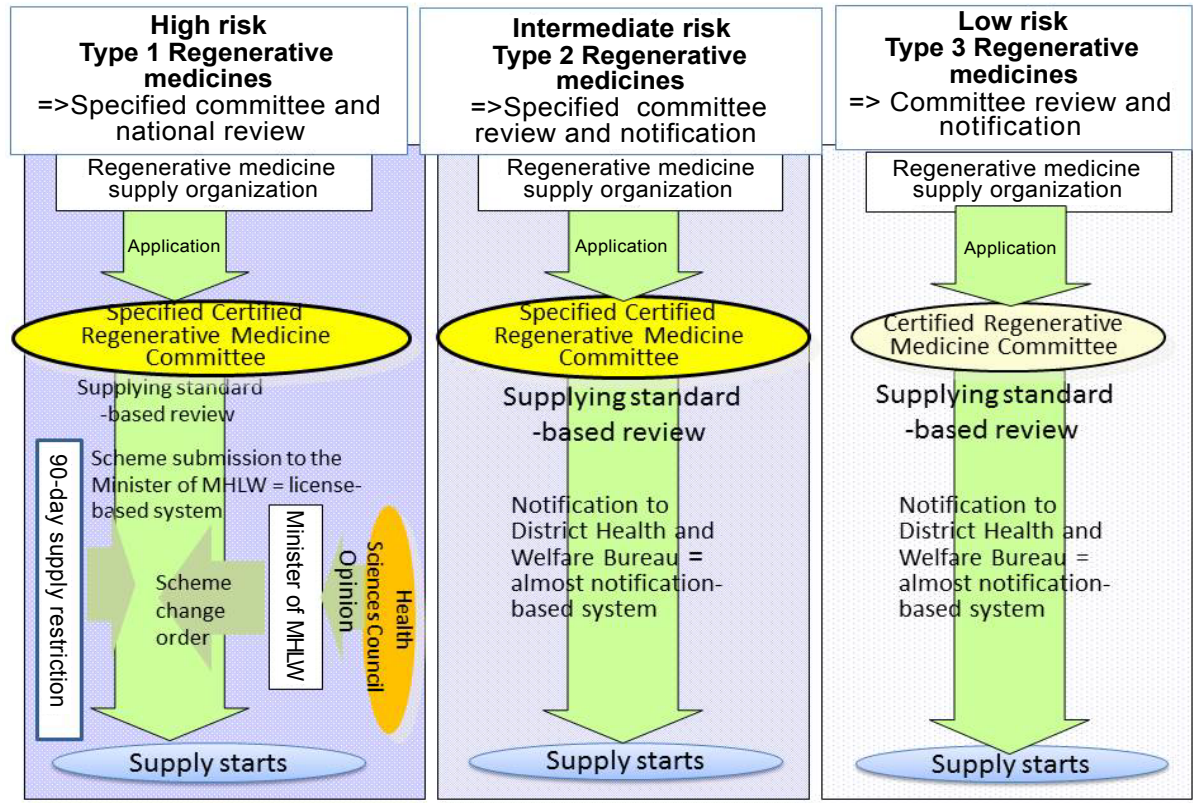

Figure 2: Risk-based review for providing regenerative medicine. The regenerative medicinal products must be reviewed before their release to the patients through the Regenerative Medicine Safety Assurance Act and there are three review-tracks according to the risk assessment.

new authority to step into the supply permitted by the medical service law reflects the fatalities from conventional non-insurance medical treatment.

To manufacture cell preparations (defined as specified cell processing products on the legal term), article 42 of the law specifies that the structures and facilities of the cell culture processing sites must satisfy the standards defined by the relevant ministerial ordinance, clearly stating that the standards of the structures and facilities for manufacturing are consigned in the ordinance (article 42 standard).
Article 44 of the law stipulates that the Health Minister can define in a ministerial ordinance the methods of manufacturing and quality control, conducting study inspections, storage and transport, and all other issues on specified cell processing products at cell culturing processing sites that manufacturing businesses of specified cell processing products should adhere to in their operations, clearly specifying that adherences by the manufacturing business of specific cell processing products should be defined by an ordinance (article 44 standard). These standards are almost on the equivalent level as the directives on human stem cell clinical research, and thus the 
enforcement of the new law does not cause any additional burden on research organizations and medical institutions that conduct clinical research on human stem cells in compliance with the directives. Meanwhile, since the cost of the equipment and maintenance of these cell processing sites is prohibitive, the Regenerative Medicine Safety Assurance Act allows outsourcing of the manufacturing of specified cell processing products. This idea stemmed from the presumption that outsourcing to an external organization with manufacturing capability would provide patients with specified cell processing products of a more stable quality. The levels required for contract manufacturing organizations inevitably became highly demanding, and notably in view of the legal framework, the law provides medical institutions with a notification system; but it also imposes a licensing system on contract manufacturing organizations. Furthermore, when a medical institution outsources the manufacturing of a specified cell processing product, the outsourcing has to be made to a licensee or a reported business. For licensing to the manufacturing site of a specified cell processing product, it is expected that discussions on the results of the law in practice may dictate the method of review and the required level of such review, but from the prediction that the Pharmaceutical Products Medical Device Agency (PMDA) would provide reviews, then it should be presumed that the level required could be equivalent to the standard for manufacturing sites in PAL. To outsource the manufacturing of a specified cell processing product, such products are administered by medical practitioners, and thus all the responsibilities are attributed to medical practitioners that administer the regenerative products, as well as the responsible managers of medical institutions, meaning that unless a defect of such specified cell processing product is demonstrated, the practitioner who administers the product is bound to bear all the civil and criminal responsibilities. Moreover, it is predictable that some corporate entities may suggest that medical practitioners at medical institution undertaking the production of regenerative medicines avoid PAL restrictions, but the PAL regulation applies to a system where the manufacturing business practically distributes their own products $[20,21]$.

\section{Advanced medical care as an exit of regenerative medicine safety assurance act}

There is concern that regenerative medicines may not be permeated through the general public as medical care via the medical service law/ medical practitioner act tracks. Meanwhile, some medical researchers express their views that it would be dauntingly challenging to promote regenerative medicines through pharmaceutical (medical device) law tracks. With this background, discussion must be essential to support a system to be designed and managed to connect these two tracks. It should connect two systems between the tracks of Regenerative Medicine Safety Assurance Act and of PAL. This is advanced medical care.

Advanced medical care is a new system established in April 2008, which succeeded its predecessor; the advanced medical care evaluation system (obsolete system), in a manner of expanded verification in clinical use and included as the third category advanced medical care within the evaluation system (Notification 0331021 by the head of the Secretary-General of Health Policy Bureau, MHLW dated March $31,2009)$. In relation to the abolishment of highly advanced medical treatments, the advanced medical care system initially excluded advanced medical technologies using unapproved or off-label pharmaceutical products/medical devices out of its scope, but as long as medical technologies as the third category of advanced medical care using unapproved or off-label pharmaceutical products or medical devices should be positively recognized as advanced medical care and the data obtained shall meet the cGCP level, then the system allows the use of data on unapproved or off-label pharmaceutical products/ medical devices in application documents for clinical trial notifications. This system envisages bridging between advanced medical care under regulation of the medical practitioner act track to the PAL track through a tool of data collection for evaluation. Meanwhile, the advanced medical care evaluation system is essentially designed for bridging developments under PAL, and the stagnancy in finding companies that undertake medical technologies intended for orphan diseases, which frequently results in a deadlock. Moreover, discussions evolved over the hierarchies in the review of the advanced medical care evaluation system and advanced medical care. Consequently, advanced medical care in the second and third categories was organized into the Advanced Medical Care [11].

In view of the quality and safety assurance and relief efforts for aftereffects/adverse drug reactions, the pharmaceutical (medical device) law track can offer some benefits to facilitate relief, whereas in view of the possibility of clinical protocol improvement, the use of the Regenerative Medicine Safety Assurance Act may deserve considerations as the law may offer more freedom with ample area of discretion left for medical practitioners. Hence, the proactive use of this system may render medical services provided outside the PAL appropriately useful for the society.

When some clinical research is completed in accordance with the Regenerative Medicine Safety Assurance Act, and researchers conclude that certain levels of satisfactory safety/efficacy are achieved, evaluations will take place to decide whether such therapy protocols should be covered by the national health insurance reimbursement program to ensure effective supply of adequate medical services. Specifically, if such clinical research protocols for medical treatments deemed evaluable and reviewed by the MHLW Advanced Medical Care Evaluation Committee to decide whether certain levels of efficacy/ safety are rationally achieved scientifically and recognized as advanced medical care, basic issues can be claimed under benefits for noninsurance mixed medical care service expenses such as hospital fees. These specified medical care coverage systems are established with the intent to respond to the sophistication of medical services associated with improved levels of life and diversified values among the public and astounding advancement of medical practices and techniques and to provide adjusting tools between appropriate insurance benefits to ensure access to necessary medical services and patient choice for access to appropriate medical services. The systems serve as tools to validate whether advanced technology provided by medical practitioners within their medical institution in accordance with Medical Practitioner Act has scientific rationality to commercialize as health insurance treatment. The hope is that all clinical research conducted in accordance with the Regenerative Medicine Safety Assurance Act will offer benefits to the society at large [1-4].

\section{Conclusions}

In previous attempts for wider distribution of regenerative medicines through development under pharmaceutical affairs regulations, the conventional PAL dealt with only two categories, pharmaceutical products and medical devices. Regenerative medicines have been the source of confusion for academia as the products could not be sterilized and thus could not be handled as pharmaceutical products, while the products cannot fully fit within the conventional regulatory contexts for pharmaceutical products. Academia had to face patients under such a regulatory system with gloomy uncertainties 
Citation: Okura H, Matsuyama A (2017) History of Development and Regulations for Regenerative Medicines in Japan. J Stem Cell Res Ther 6: 372. doi: 10.4172/2157-7633.1000372

without indicators or lights to clearly show the directions. The question to such uncertainties led to upgrading of directives for human stem cellbased clinical research to the Regenerative Medicine Safety Assurance Act and the clear establishment of regenerative medicine products as a new category in connection with enactment of the PMD Act, the latest revised PAL. With the revision, two paths became available for societal contribution of regenerative medicines. Japan is the only country among the developed countries where society can enjoy these two paths, and social experiments on regenerative medicines in spectacular proportion are launched for societal contribution in the world's exciting arena. The choice of either of the paths will serve as an answer to the question as to how regenerative medicines should be permeated through the society.

\section{References}

1. Laws for Promotion of Comprehensive Measures to Facilitate Swift Distribution and Safe Use of Regenerative Medicines.

2. Regenerative Medicine Safety Assurance Act.

3. Pharmaceutical and Medical Device Act.

4. Medical Innovation

5. Okano H (2014) Stem cell research and regenerative medicine in 2014: firs year of regenerative medicine in Japan. Stem Cells Dev 23: 2127-2128. [PubMed]

6. Egawa K (2004) Immuno-cell therapy of cancer in Japan. Anticancer Res 24 3321-3326. [PubMed]

7. Egawa K (2004) On the safety assurance of cell processing carried out in medical institutions for autologous immuno-cell therapy. Hum Cell 17: 1-6.

8. Sipp D (2015) Conditional approval: Japan lowers the bar for regenerative medicine products. Cell Stem Cell 16: 353-356. [PubMed]

9. Ikka T, Fujita M, Yashiro Y, Ikegaya H (2015) Recent Court Ruling in Japan Exemplifies Another Layer of Regulation for Regenerative Therapy. Cell Stem Cell 17: 507-508. [PubMed]

10. Saito M (2016) Current Status of Community Pharmacies: Expectations as a Health Information Hub, the Enforcement of Revised Pharmaceutical and
Medical Device Act, and a New Role as Stakeholders. Yakugaku Zasshi 136 245-249. [PubMed]

11. Masuyama K, Isobe S (2010) Social change and Pharmaceutical Affairs Law (PAL). Yakushigaku Zasshi 45: 78-81. [PubMed]

12. Fujiwara Y, Kobayashi K (2002) Oncology drug clinical development and approval in Japan: the role of the pharmaceuticals and medical devices evaluation center (PMDEC). Crit Rev Oncol Hematol 42: 145-155. [PubMed]

13. Nakada T, Ikeda D, Yokota M, Kawahara K (2010) Analysis of the costeffectiveness of remifentanil-based general anesthesia: a survey of clinical economics under the Japanese health care system. J Anesth 24: 832-837. [PubMed]

14. Hayakawa T, Aoi T, Umezaw A, Ozaw K, Sato Y, et al. (2015) Study on ensuring the quality and safety of pharmaceuticals and medical devices derived from processing of autologous human somatic stem cells. Regenerative Therapy 1 : 57-69. [PubMed]

15. Hayakawa T, Aoi T, Umezaw A, Ozaw K, Sato Y, et al. (2015) Study on ensuring the quality and safety of pharmaceuticals and medical devices derived from processing of allogenic human somatic stem cells. Regenerative Therapy 1: 70-80. [PubMed]

16. Hayakawa T, Aoi T, Umezaw A, Ozaw K, Sato Y, et al. (2015) Study on ensuring the quality and safety of pharmaceuticals and medical devices derived from processing of autologous human induced pluripotent stem (-like) cells. Regenerative Therapy 1: 81-94. [PubMed]

17. Hayakawa T, Aoi T, Umezaw A, Ozaw K, Sato Y, et al. (2015) Study on ensuring the quality and safety of pharmaceuticals and medical devices derived from processing of allogenic human induced pluripotent stem (-like) cells. Regenerative Therapy 1: 95-108. [PubMed]

18. Hayakawa T, Aoi T, Umezaw A, Ozaw K, Sato Y, et al. (2015) Study on ensuring the quality and safety of pharmaceuticals and medical devices derived from processing of human embryonic stem (-like) cells. Regenerative Therapy 1:109-122. [PubMed]

19. Japanese law translation design act.

20. Japanese law translation Medical Care Act.

21. Drabiak-Syed K (2013) Challenging the FDA's authority to regulate autologous adult stem cells for therapeutic use: Celltex therapeutics' partnership with RNL Bio, substantial medical risks, and the implications of United States v. Regenerative Sciences. Health Matrix Clevel 23: 493-535. [PubMed] 